Vol. 70, N. ${ }^{\circ}$ II2 (noviembre 20I8), I3-3I

\title{
¿SUSTENTABILIDAD DEL SISTEMA AGROALIMENTARIO ECUATORIANO? UNA APROXIMACIÓN A LAS ESTRATEGIAS DE PODER QUE PRODUCEN SUS PATRONES METABÓLICOS
}

\author{
GEOVANNA LASSO GONZÁLEZ \\ Universidad Autónoma de Barcelona (España) \\ Colectivo Agroecológico del Ecuador \\ Recepción de manuscrito: 17 de septiembre de 2018 \\ Aceptación versión final: 26 de octubre de 2018
}

\begin{abstract}
RESUMEN La sustentabilidad del sistema agroalimentario (SAG) y la soberanía alimentaria (SA) requieren de un diagnóstico integral para plantear estrategias realistas e informadas que las viabilicen. La economía ecológica y la ecología política ofrecen un marco teórico-analítico que favorece este análisis gracias a su carácter multidisciplinar. Bajo esta mirada se determinó que los patrones metabólicos del SAG ecuatoriano son producidos por el modelo capitalista, cuyas estrategias y redes de poder trascienden la institucionalidad pública y favorecen la acumulación de los grupos económicos que lideran el sector. Este análisis evidencia la insustentabilidad del SAG y el riesgo que corre la sA de continuar con el patrón actual.
\end{abstract}

PALABRAS CLAVE Sistema agroalimentario, soberanía alimentaria, sustentabilidad, metabolismo, estrategias de poder.

ABSTRACT The sustainability of the agro-alimentary system (AFS) and food sovereignty (FS) require a comprehensive diagnosis to rise realistic and informed strategies that allow their viability. The ecological economy and the political ecology offer a theoretical-analytical framework that favours this analysis thanks to its multidisciplinary character. Under this view it was determined that the metabolic patterns of the Ecuadorian AFs are produced by the capitalist model, which power strategies and networks transcend the public institutionality and favour the capital accumulation of the economic groups that lead the sector. This analysis demonstrates the unsustainability of AFS and the risk that FS affront to continue with the current pattern.

KEYWORDS Agrifood system, food sovereignty, sustainability, metabolism, power strategies.

JEL CODES Q150, Q160, Q180.

\section{INTRODUCCIÓN}

La Constitución del Ecuador reconoce la soberanía alimentaria (sA) como objetivo estratégico, sin embargo, la dirección de la política pública ha favorecido mayoritariamente la expansión de un modelo de producción que favorece la expansión de los commodities ${ }^{1}$ para la exportación y la industria, poniendo en riesgo la consecución de este objetivo. 
Generar política pública que procure la sustentabilidad del sistema agroalimentario y la SA requiere de una comprensión integral del estado de este sistema. Explorar cuáles son las limitaciones biofísicas, económicas y sociales es un paso importante y — se podría decir- esencial en este proceso, cuando la voluntad política está orientada a garantizar el cumplimiento del objetivo de la Constitución, a privilegiar al ser humano y la naturaleza sobre el capital y los intereses económicos. Sin embargo, esté análisis no sería suficiente, dado que atrás de la expansión del modelo capitalista en el agro se encuentran también los intereses de los grupos económicos que pretenden concentrar los distintos sectores del sistema agroalimentario.

En esta línea, es imprescindible utilizar un marco multidisciplinar e interdisciplinar, que permita por un lado este diagnóstico integral del sistema agroalimentario, abordando su multiescalaridad y multidimensionalidad. Para esto, es preciso asumirlo como un sistema interdependiente del sistema ecológico y social y explorar el metabolismo social y ecológico que lo caracteriza. Por otro lado, y con el fin de generar un marco de análisis realista sobre el cual plantear las diferentes estrategias necesarias para garantizar la sustentabilidad y la sA, es necesario entender cuáles son las causas estructurales de la ruptura metabólica que caracteriza a los sistemas socioecológicos en la actualidad, de la conflictividad social y ambiental que ésta genera, y de los procesos de exclusión social y degradación ambiental actuales.

La economía ecológica y la ecología política constituyen una herramienta teórica y analítica para realizar este acercamiento integral al sistema agroalimentario, evaluar su estado, y plantear una política pública que viabilice su sustentabilidad. Al mismo tiempo, permite identificar las limitaciones de la institucionalidad y la política pública, y plantear estrategias informadas y realistas que procuren un modelo alternativo que favorezca la consecución de la sA.

Con este contexto, los objetivos de este artículo son: 1. exponer la importancia de incorporar en el diagnóstico, la toma de decisiones, la elaboración de la política pública, los procesos autónomos y militantes, los marcos de análisis que ofrece la ecología política y la economía ecológica; 2. presentar un análisis, con este enfoque, de los actores y algunas de las estrategias de poder que permiten la expansión del modelo capitalista en el sistema agroalimentario ecuatoriano; y 3. analizar la evolución de los fondos y flujos en el sector interno y externo que caracterizan el metabolismo del sistema agroalimentario actual en el Ecuador como indicadores de su sustentabilidad y de la viabilidad de la soberanía alimentaria.

\section{ECONOMÍA ECOLÓGICA Y ECOLOGÍA POLÍTICA PARA UN DIAGNÓSTICO INTEGRAL DEL SISTEMA AGROALIMENTARIO Y EL PLANTEAMIENTO DE ESTRATEGIAS REALISTAS PARA PROCURAR SU SUSTENTABILIDAD}

La economía ecológica surge de la necesidad de salir de la lógica crematística y conservadora de la economía convencional para poder entender de manera más integral las implicaciones que tiene el sistema económico sobre el equilibrio ambiental y la justicia social, reconociendo y entendiendo los límites biofísicos del ecosistema mundo, y los conflictos socioambientales que desencadena el no contemplarlos (Martínez Alier, 1999; Estevan, Jover y Naredo, 2009; Herrero, 2015). La multidiciplinariedad que recoge ha sido esencial en esta comprensión integral y más amplia del sistema económico y su relación con los sistemas ambientales y sociales 
(Georgescu-Roegen, 2007). La economía ecológica, bioeconomía o economía humana debería, de acuerdo a Gerogescu-Roegen (2007), tener la finalidad de:

...[A]dministr(ar) los recursos y $\log ($ ar) un control racional sobre el desarrollo y las aplicaciones tecnológicas, de modo que sirvan a las necesidades humanas reales (...) una economía de la supervivencia, o mejor aún, de la esperanza (...) basada en la justicia, que haga posible la distribución equitativa de la riqueza de la Tierra entre la población, tanto actual como futura. (p. 33)

Este entendimiento permitiría plantear modelos alternativos que prioricen la sustentabilidad ambiental, el bienestar humano y otras formas de relacionamiento de la sociedad que privilegien la solidaridad y los cuidados, y poniendo en un segundo plano el crecimiento económico (Herrero, 2015; Estevan, et al., 2009).

Una de las premisas básicas de la economía ecológica es entender que el sistema económico no es un sistema cerrado, más bien es parte del sistema ecológico en el cual está inmerso, con el cual intercambia materiales y energía (Georgescu-Roegen, 2007; Van Hauwermeiren, 1999). Esta comprensión nos lleva a dos conceptos vinculados entre sí: metabolismo y entropía. Al igual que un ser vivo, una sociedad, dependiendo de su sistema económico y modelo productivo, posee un metabolismo que demanda más o menos materiales y energía y que produce más o menos residuos. En este marco, la economía ecológica permite evidenciar que los materiales y energía que se utilizan dentro del sistema económico tienen un antes y un después de haber ingresado en su ciclo, generan por un lado residuos y por otro una cantidad de energía y materiales que no puede volver a ser utilizada en el ciclo productivo, entrando en un proceso entrópico (Georgescu-Roegen, 2007). De acuerdo a la naturaleza, rol y permanencia o no en el sistema analizado, existen tres tipos de recursos. Los stocks son recursos finitos que pueden agotarse durante el tiempo de análisis del sistema estudiado, como el petróleo, los minerales o la fertilidad del suelo (Giampetro y Lomas, 2014). Aquellos recursos que se utilizan, pero permanecen «iguales» a lo largo del proceso productivo se les denominan fondos. En el caso del sistema agroalimentario, sus fondos críticos son la tierra, campesinado y productores en general; el capital tecnológico y la maquinaria son otros fondos de los sistemas socioeconómicos (Georgescu-Roegen, 2007; Giampetro y Lomas, 2014). Finalmente, aquellos recursos que durante el proceso productivo aparecen y desaparecen durante el análisis se denominan flujos. La electricidad, alimentos, agua de riego e insumos externos utilizados en agricultura son ejemplos de flujos (ibid.). La sustentabilidad de determinado sistema depende principalmente de la gestión y uso de los stocks y la intensidad de uso de sus flujos.

El análisis de la sustentabilidad del sistema agroalimentario requiere conocer: i. el uso y destino de sus fondos críticos: la tierra y la mano de obra campesina, ii. la intensidad de su metabolismo en cuanto a consumo de materiales y energías, la cual varía de acuerdo a los modelos productivos y su dependencia a insumos externos (Giampetro y Lomas, 2014), y iii. el flujo de alimentos en el sector externo, es decir, la cantidad de alimentos que es exportada e importada; mientras que en el sector interno: a qué se destina el suministro de alimentos a nivel nacional. En el marco de la soberanía alimentaria, es necesario complementar este análisis preguntándose también qué actores están a cargo de esta producción, qué se está produciendo, cómo y con qué fin se está produciendo y qué alimentos componen la dieta de la población. 
Si bien hasta aquí este análisis nos permite evaluar la sustentabilidad del sistema agroalimentario, queda un vacío en cuanto al entendimiento de cuáles son los modelos de sistemas productivos y económicos, los actores y las estrategias de poder que permiten producir y reproducir los diferentes metabolismos socioeconómicos. Es aquí donde la mirada que brinda la ecología política, desde su multidisciplinariedad, permite ampliar, profundizar y complejizar el análisis de la economía ecológica. Comprender qué conflictos socioambientales y de distribución desigual se generan, quiénes están detrás de las inequidades y desigualdades, del despojo, de la degradación ambiental. Cuáles son los mecanismos que permiten colocar a la naturaleza y al ser humano al servicio de la acumulación de pocos actores. Reconocer los procesos de colonización y mercantilización de la naturaleza y de la vida en general, y las diversas estrategias utilizadas, muchas veces bajo discursos de desarrollo sostenible o eficiencia, como se observa en las propuestas de capitalismo verde.

La ecología política reconoce que es el sistema capitalista imperante a nivel global el que fomenta un modelo agroalimentario basado en la modernización, el libre mercado global, la financiarización de la agricultura, el consumismo individualista y el desperdicio masivo; y que es este modelo el que viabiliza la acumulación de las élites empresariales a nivel nacional y las transnacionales al nivel internacional (Bernstein, 2016; McMichael, 2009, 2012; Borras, Franco, Gómez, Kay y Spoor, 2012). Su marco de análisis demanda identificar los actores que ejercen este poder y las estrategias que utilizan para expandir este modelo capitalista, así como entender cuál es el rol que ocupan en el sistema agroalimentario, sus nexos con el Estado, el sistema financiero y demás actores que lo lideran a nivel nacional e internacional. Considera igual de necesario identificar a las clases subalternas oprimidas que sufren las inequidades e injusticias del sistema; reconocer y valorar los diferentes procesos y estrategias individuales y colectivas que les permiten resistir o construir otras formas de producir y relacionarse entre sí y con la naturaleza, otros modelos alternativos al capitalismo.

Lo hasta aquí expuesto evidencia la necesidad de la mirada conjunta de la economía ecológica y de la ecología política, no solo para la comprensión integral y estructural de determinado sistema socioeconómico, sino para mirar con claridad la limitación de la institucionalidad y de las políticas públicas, y plantear estrategias realistas para procurar modelos alternativos sostenibles en el campo agroalimentario, productivo y socio-económico en general. En este caso, un análisis integral de la sustentabilidad del sistema agroalimentario y la viabilidad de la soberanía alimentaria, requiere integrar al análisis de su metabolismo los aspectos antes mencionados.

En el intento de complementar los conocimientos en torno al estado del sistema agroalimentario del Ecuador desde esta perspectiva, a continuación se presentan unos rasgos generales sobre los principales actores y las relaciones de poder que fomentan el modelo capitalista en el agro ecuatoriano, la evolución y estado actual del metabolismo del sistema agroalimentario y sus implicaciones en la soberanía alimentaria.

\section{MODELO CAPITALISTA Y ACUMULACIÓN: ACTORES, ESTRATEGIAS Y REDES DE PODER}

En el Ecuador, las élites que gobiernan el sector agroalimentario se vienen capitalizando desde inicios de siglo. Su acumulación originaria surge a partir de la producción, industrialización 

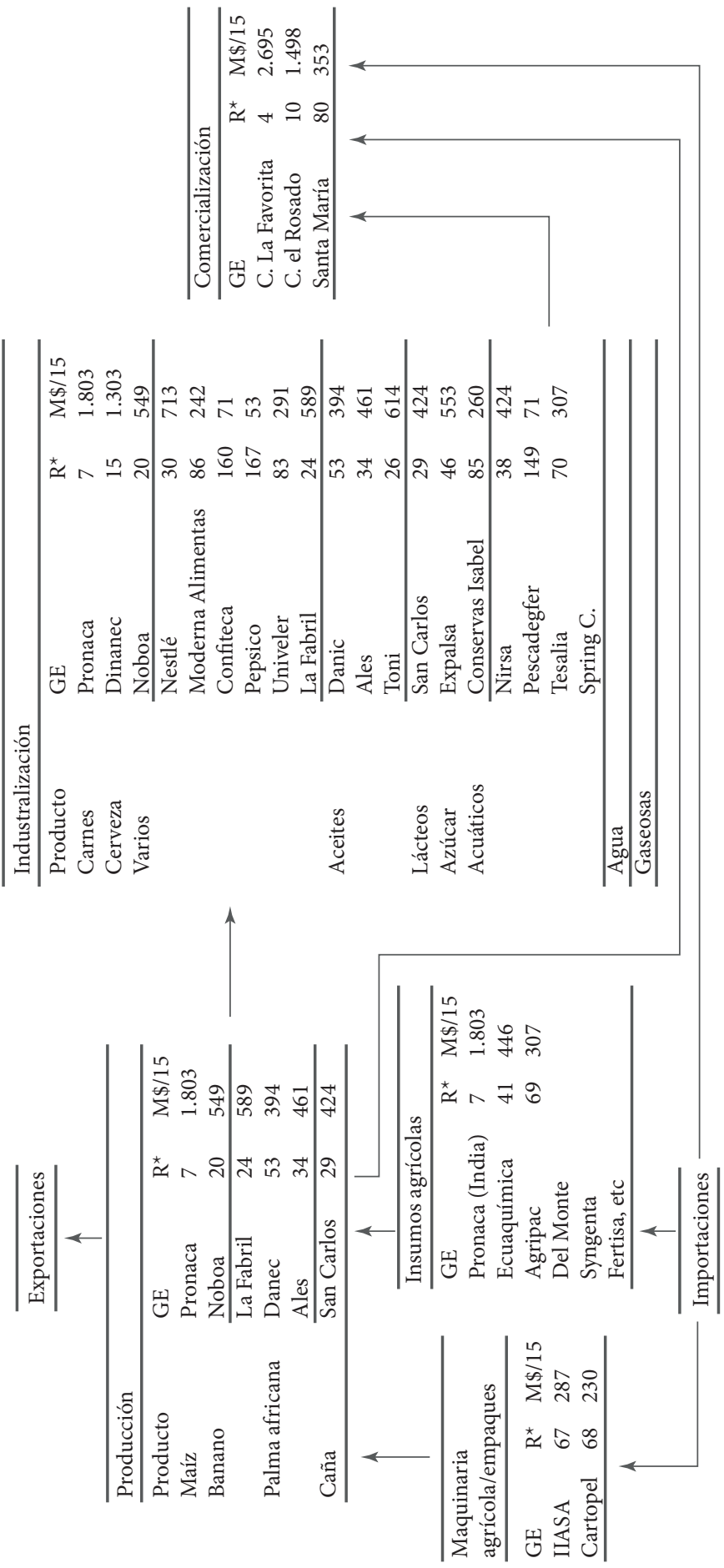

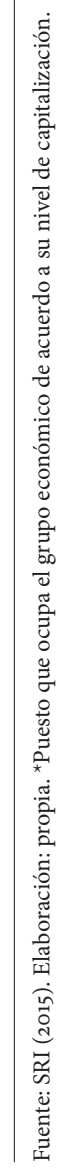


y/o exportación de commodities como el cacao, banano, café, posteriormente palma africana y productos del mar. Otro grupo levanta su capital en base a importación y comercialización de productos vinculados con la producción y el consumo, concentrando el mercado a nivel nacional. Su configuración se basa principalmente en lazos familiares, apoyados por vinculaciones políticas y financieras (Navarro, 1976; citado en Pastor, 2016), mientras que el proceso de monopolización se facilita gracias a una integración vertical - toda la cadena - y horizontal - una empresa maneja varios productos del mismo bien - , a la diversificación — conformación de varias empresas - , y formación de conglomerados — unión de empresas de varios sectores- (CEDIS, 1986; citado en Pastor, 2016). Así, varios grupos económicos que manejan el sector agroproductivo, la agroexportación, la producción y comercialización de alimentos, también acaparan otras ramas productivas, industriales y comerciales y tienen acciones en el sector financiero y medios de comunicación.

En la figura 1 se pueden observar los principales grupos económicos que lideran los distintos sectores en el ámbito agroalimentario, y los flujos económicos y materiales en el sector interno - producción y comercialización interna- y externo -importación y exportación-. Se puede ver que algunos de los grupos económicos se repiten en los diferentes sectores, y que algunos de ellos se encuentran entre los 20 principales grupos económicos del país de acuerdo a su nivel de capitalización.

Estos grupos se constituyen de capitales nacionales y extranjeros, otra estrategia importante para capitalizar y acumular. Por citar algunos ejemplos, el Grupo Noboa está conformado por 183 miembros, de los cuales 6 son personas naturales extranjeras, 13 sociedades extranjeras y 5 instituciones financieras. El Grupo Pronaca tiene 156 miembros, de los cuales 2 son personas naturales extranjeras y 24 son sociedades extranjeras. En el grupo El Rosado, de los 68 miembros, 4 son personas naturales extranjeras, 27 sociedades extranjeras y 3 medios de comunicación.

Estas élites económicas - junto con el Estado-, la gran mayoría de instituciones educativas, el sector financiero nacional e internacional, las corporaciones transnacionales, son algunos de los actores que tejen una red de poder interescalar que permite la expansión del modelo capitalista en el sector agroalimentario, que produce el territorio y el metabolismo que lo rige.

Las estrategias del poder son diversas. Históricamente, la presión o la vinculación directa de los grupos de poder con los gobiernos neoliberales de turno los beneficiaron a través de marcos legales, decretos, la política pública en general. Algunas de estas leyes fueron la Ley de Fomento y Desarrollo Agropecuario lanzada en 1979 por el Triunvirato León-Poveda-Franco ante las presiones directas de latifundistas y Cámaras de la Agricultura y Ganadería; la Ley de Desarrollo Agrario firmada en 1994 en el gobierno de Sixto Durán Ballén, la cual elimina la Reforma Agraria de 1973 y garantiza los derechos y privilegios de los latifundistas, promociona el mercado de tierras, entre otras (Zapata, Ruiz y Brassel, 2008). Otra consecuencia negativa de estas leyes para las luchas campesinas fue la reducción de la reforma agraria a la titulación de tierras, lo cual se mantuvo durante el gobierno de Rafael Correa y del actual Lenín Moreno.

La formulación de decretos también ha sido otro mecanismo. Por ejemplo, el presidente Gustavo Noboa Bejarano - parte del grupo Noboa Bejarano, dueño en ese entonces de Aceites La Favorita-, mediante decreto $2961,{ }^{2}$ cambia la categoría de uso del suelo en San Lorenzo, de 


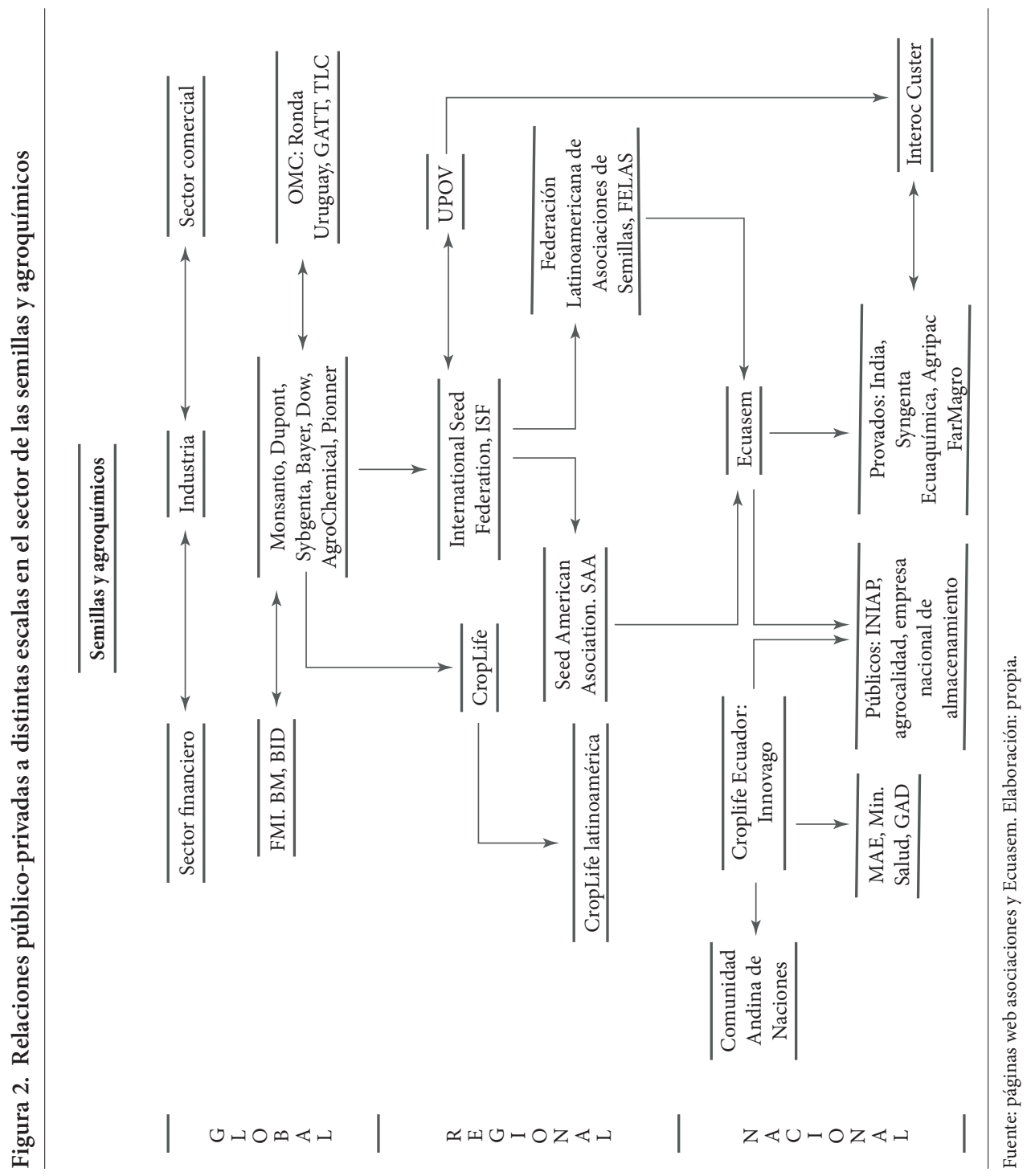

uso forestal a tierra agrícola, para validar la permanencia y expansión de las empresas palmicultoras en la zona: Palmeras de los Andes perteneciente a DANEC y Palmeras del Pacífico perteneciente a La Favorita (Buitrón, 2001). Otro ejemplo es la concesión de 20.000 hectáreas de bosque primario en la Amazonía ecuatoriana por parte del Instituto Ecuatoriano de Reforma Agraria a dos grandes empresas de palma africana en 1979 (Ashley, 2010).

En el gobierno de Rafael Correa, los grupos económicos tradicionales dejan de ser parte y no se encuentran vinculados directamente con el Gobierno, sin embargo, otra estrategia de poder continuó beneficiándolos a través de la política pública: la relación saber-poder-discurso. 
No existe relación de poder que no se corresponda a un campo del conocimiento, ni existe conocimiento que no se corresponda con una relación de poder (Foucault, 2014). En el campo agroalimentario se pueden evidenciar varios de los postulados de Foucault respecto a la relación entre conocimiento y poder, al carácter positivo del poder como estrategia de dominación, y cómo, en su conjunto, las distintas estrategias de poder permiten el control de diversos ámbitos de la sociedad, determinan los distintos espacios y esferas de la vida de los individuos, y a través de la práctica productiva afectan a la naturaleza, constituyéndose así en una forma de biopoder.

El conocimiento que se viene estableciendo como verdad desde los 60 se ha ido consolidando tanto a nivel de problemáticas como de soluciones, y ha ido calando en un amplio sector y diversos actores vinculados con el sistema agroalimentario. El hambre en el mundo se debe a un problema de productividad; la baja productividad es producto del uso de semilla de mala calidad, la poca tecnificación y la prevalencia de las prácticas atrasadas e ineficientes de la agricultura campesina; la pobreza de los pequeños y medianos productores es producto de esta baja productividad y de la escasa vinculación con los mercados nacionales e internacionales. Establecidas estructuralmente estas problemáticas-verdades, es más fácil presentar a la modernización de la agricultura como la única solución ante el problema de la productividad, la vinculación al mercado internacional como motor del crecimiento económico, y a la empresa privada y su innovación como motor económico y generadora de empleo. El uso de la semilla certificada y transgénica, la tecnificación y maquinización, el encadenamiento productivo de commodities, serían entonces estas soluciones-verdades, muchas veces manejadas desde el discurso del capitalismo verde, planteado como una nueva estrategia de acumulación con rostro sostenible y social. Estas verdades no solo aceptadas por la ciencia, los técnicos, las autoridades, productores y parte del campesinado, sino solicitadas y puestas en práctica por todos estos actores. Este cocimiento hegemónico se difunde a través de las instituciones educativas, generando un ejército de técnicos, burócratas y autoridades que plasman este conocimiento en el territorio a través de la ejecución de la política pública, los proyectos de desarrollo rural, local, comunitario, agroproductivo, etc.

Las redes de lobbies, corrupción y vinculaciones políticas, estructuralmente implantadas en áreas clave de algunas instituciones públicas, constituye otra estrategia que complementa la red de poder. Las empresas vinculadas con las semillas y agroquímicos, por ejemplo, se valen de esta estrategia para favorecer su acumulación, como se puede identificar en áreas clave del Ministerio de Agricultura (MAG). De acuerdo a la información obtenida a través de informantes clave, en la Subsecretaría de Comercialización y en la Dirección de Semillas y Agrobiodiversidad trabajaron personas vinculadas con la industria de los agroquímicos, las cuales jugaron un rol clave en la elaboración y ejecución del Proyecto de Semillas Mejoradas para Cadenas Estratégicas (PSMCD, detallada más adelante) (Informantes 1 y 2). La presión que ejercen los sectores ganaderos y bananeros en las decisiones que toma este ministerio dirige también el diseño y ejecución de la política pública (Informante 3).

Estas estrategias de poder trascienden las escalas y las fronteras, creando una verdadera red de poder. Esta geometría de poder (Massey, 1993) se puede observar claramente en la implantación del modelo modernizador basado en el kit tecnológico, a través de la creación 


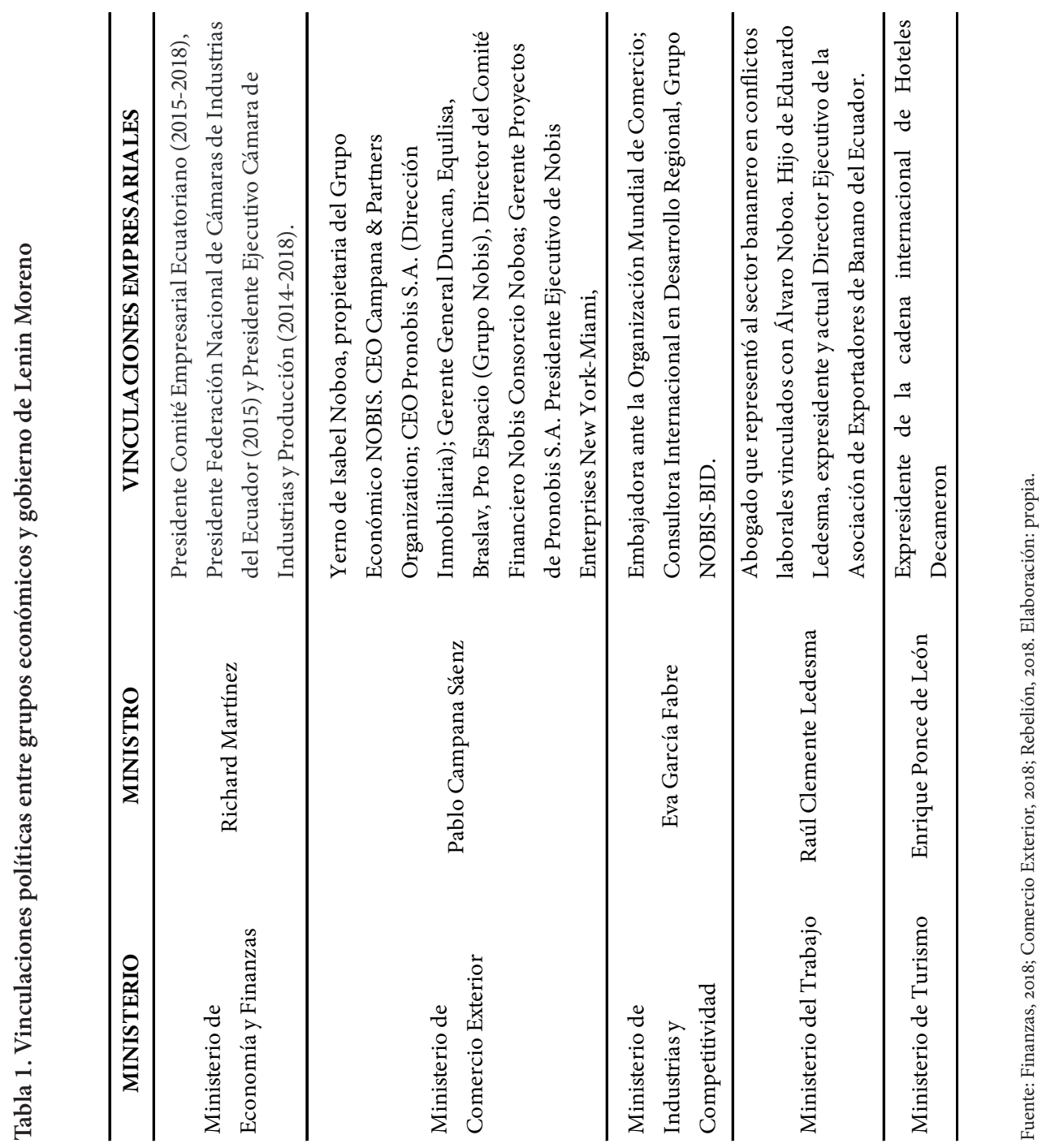

de una interfase institucional que vincula al Estado con el sector privado. Es el caso de la Asociación Ecuatoriana de Semillas, Ecuasem, conformada por instituciones públicas - INIAP, Agrocalidad, unA - y privadas - Agripac, Ecuaquímica, India/Pronaca, Interoc-Custer, Fram Agro, Syngenta, Monsemillas, Semimor-. Ecuasem tiene el objetivo de promocionar el uso de la semilla certificada en el país a través de distintos mecanismos, y de procurar marcos legales favorables tanto para el incentivo en el uso, como sucedió en el proceso de construcción de la Ley de Semillas y Agrobiodiversidad (Informante 4), como en temas de propiedad intelectual. Esta organización es parte de una red de organizaciones que funcionan en los niveles nacional, regional e internacional (ver Figura 2), y que representan los intereses de la 
industria de la semilla y los agroquímicos a nivel global. Al nivel regional, Ecuasem se enlaza con la Seed American Asociation (SAA) y la Federación Latinoamericana de Asociaciones de Semillas (FELAS); a nivel internacional, es la Federación Internacional de Semillas (ISF por sus siglas en inglés), la cual trabaja desde 1923. Al igual que Ecuasem, en Ecuador existen organizaciones representantes de la industria en el resto de países de la región. El objetivo común es expandir el uso de la semilla certificada, para lo cual actúan en varios espacios para fomentar el uso, el libre comercio, marcos regulatorios favorables para temas de propiedad intelectual, salud y ambiente.

CropLife es otra organización que representa a las mismas industrias y que trabaja a nivel global, regional (CropLife Latino América) y nacional como CropLife Ecuador-InnovAgro, promoviendo el manejo adecuado de agroquímicos y de los envases, para lo cual trabaja de la mano con instancias del Ministerio del Ambiente y el de Agricultura; participa también en todos los espacios de regulación y legislación posibles.

Si bien esta estrategia de poder sigue su curso al estar estructuralmente integrada en la institucionalidad pública y privada vinculada con el sector agroalimentario, los grupos económicos de este sector se han visto beneficiados nuevamente por la vinculación directa con el gobierno de Lenín Moreno, quien ha colocado en ministerios estratégicos a representantes de estas élites (ver Tabla 1). A menos de un año y medio de gobierno estos grupos se han visto beneficiados no solo a través de la continuidad de la política pública que ya los beneficiaba, sino también con nuevas políticas que los beneficia directamente. Algunos ejemplos son la firma de nuevos acuerdos comerciales en búsqueda de ampliar el mercado de sus productos; la condonación de la deuda millonaria que estos grupos tenían con el Estado por evasión fiscal; la supresión del pago del adelanto al impuesto a la renta, la flexibilización laboral permitida en ciertas cadenas — florícola, agrícola, acuícola, ganadería, turismo, construcción, arte-, entre otros beneficios estipulados en la Ley de Fomento Productivo (2018).

Hasta aquí se han expuesto algunos de los principales actores y estrategias de poder que permiten la expansión del modelo capitalista en el agro y la acumulación de los grupos de poder que dominan el sector. Ahora, ¿cómo se expresan estas dinámicas en el metabolismo del sector agroalimentario a nivel nacional?, ¿para qué fin están siendo destinados la tierra y la mano de obra campesina?, ¿qué tan dependientes de insumos externos es este sector?, ¿cómo ha evolucionado el flujo de alimentos externa e internamente y cómo impacta esto en la soberanía alimentaria del país? En el siguiente acápite se presentan algunos rasgos de este metabolismo creado por estos actores y dinámicas de poder.

\section{LOS FLUJOS METABÓLICOS DEL SECTOR AGROALIMENTARIO DEL ECUADOR: EVOLUCIÓN E IMPACTO EN LA SOBERANÍA ALIMENTARIA}

La tierra y la mano de obra campesina constituyen dos de los fondos esenciales del metabolismo del sistema agroalimentario. Para garantizar la soberanía alimentaria del país y la sustentabilidad del sistema agroalimentario, como lo demanda la Constitución, la política pública debería procurar que estos fondos estén orientados a la producción del alimento suficiente y culturalmente apropiado para la población ecuatoriana. Que esta producción se realice bajo 


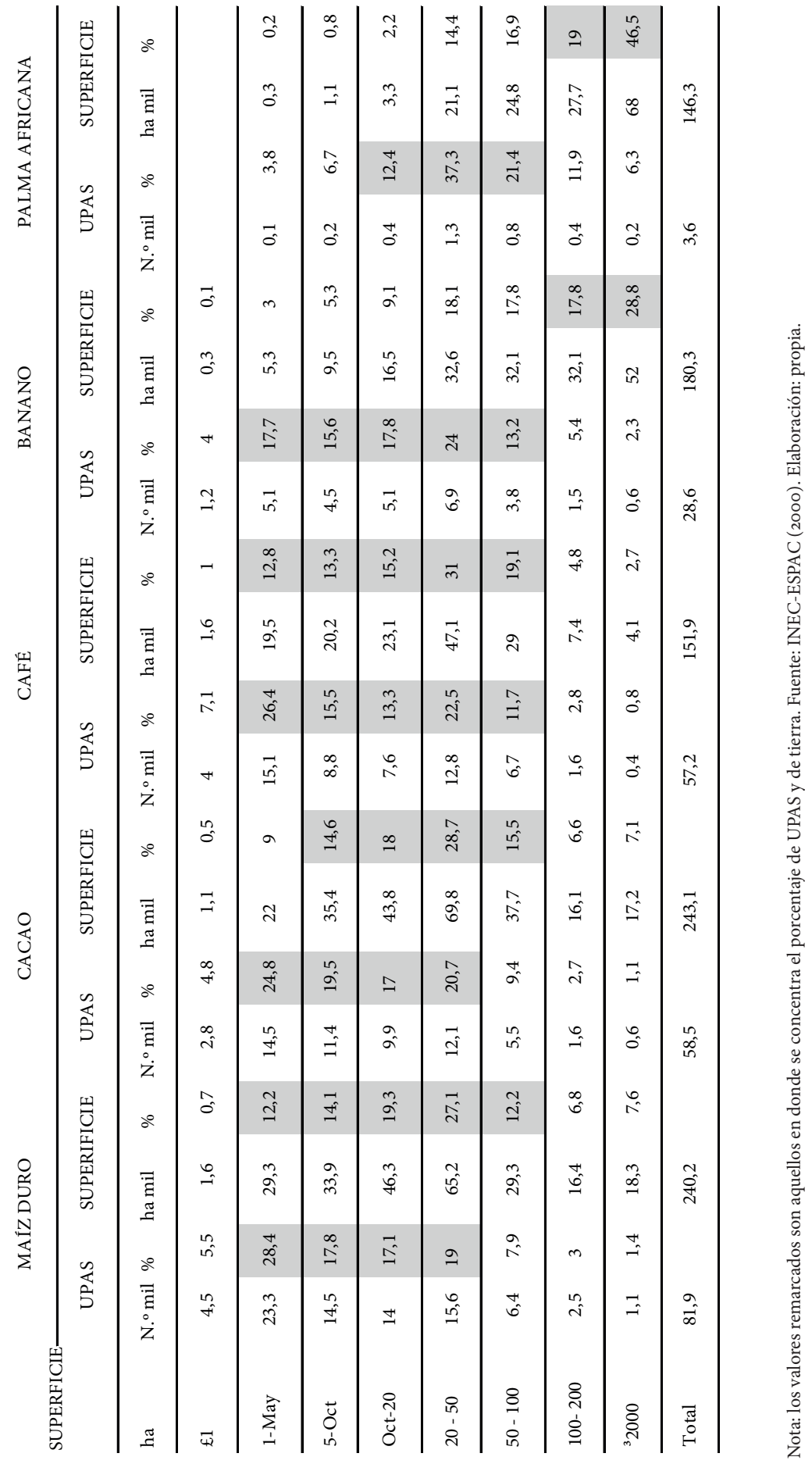


un modelo que no degrade la fertilidad del suelo ni destruya la agrobiodiversidad vinculada con la cultura de los pueblos y el ser campesino; que no genere dependencia tecnológica ni de mercado a través de kits impuestos en la lógica de modelos universales, que no enferme a productores y consumidores, entre otros aspectos. Sin embargo, la situación actual está lejos de garantizar estos mínimos que demanda un modelo basado en la soberanía alimentaria.

Si analizamos el uso actual del suelo, de acuerdo la Encuesta de Superficie y Producción Agropecuaria del Instituto Nacional de Estadística y Censos (INEC, 2014) observamos que la superficie plantada de los cultivos permanentes, semipermanentes y el maíz duro, orientados a la exportación y la industria, incrementaron 220.000 hectáreas entre el 2002 y el 2014; mientras la superficie de los cultivos transitorios, orientados al abastecimiento interno, disminuyó 174.00o hectáreas en el mismo período. Esta producción diversificada y orientada al consumo interno está relegada mayormente a suelo agrícola con fertilidad baja: el mosaico agropecuario ocupa apenas del 8,5\% de la tierra fértil con vocación agrícola, mientras los cultivos permanentes, semipermanentes ${ }^{3}$ y pastizales ocupan el $61,2 \%{ }^{4}$

La expansión de los cultivos orientados a la exportación y la industria se ha dado mayoritariamente sobre suelos destinados al mosaico agrícola, cultivos anuales y sobre bosque nativo, páramos, vegetación arbustiva y herbácea. Entre el 2008 y el 2014 la expansión del maíz duro, orientado a la industria de balanceado, ${ }^{5}$ causó la mayor parte de los cambios de uso del suelo, seguido de la palma, el cacao y el café. El 76\% del suelo agropecuario reemplazado corresponde al mosaico agrícola, seguido de cultivos anuales (13\%) y semipermanentes (11\%). El 90\% del reemplazo del mosaico agrícola también es ocasionado por la expansión del maíz, el cacao, el café y la palma - en ese orden-.

Los territorios sobre los cuales se está dando este reemplazo de cultivos, son territorios mayormente de la Costa, en donde la agroindustria se viene implantando y expandiendo desde los años 70, y en la Amazonía norte, en donde se expande principalmente la palma africana, el café y el cacao.

\section{ENCADENAMIENTOS PRODUCTIVOS Y AGRICULTURA BAJO CONTRATO:}

CAMPESINADO AL SERVICIO DEL CAPITAL

El encadenamiento productivo bajo la modalidad de agricultura bajo contrato es un modelo que consiste en la vinculación de pequeños y medianos productores a una empresa ancla a través de un contrato, el cual estipula que la empresa ancla comprará la materia prima a los productores de manera permanente, mientras ésta les proporciona asistencia técnica, facilidad de crédito, el kit tecnológico, entre otros acuerdos, dependiendo del sector. La agricultura bajo contrato con intervención estatal inicia en el año 2001 con el proyecto PROLOCAL, financiado por el Banco Mundial, el cual fomenta la organización de los productores, el registro en el Ministerio de Bienestar Social, y el anclaje a empresas como AGRIPAC a través de la firma de un contrato que estipula que la dotación de semillas y agroquímicos por parte de la empresa sería cancelada a través de la producción de maíz (Yumbla, 2014). En el Gobierno de Rafael Correa este modelo de desarrollo rural se implementó principalmente a través del Programa de Negocios Rurales Inclusivos (Proneri) en el que participan Nestlé, Pronaca, Floralp, Energypalma, Nintanga, Agroparaiso, Triari y Transmar (FAO, 2013); el Proyecto FICA que otorga crédito a través de la Corporación Financiera Nacional (CFN) para la adquisición de semillas híbridas 
Figura 3a. Importacion de agroquímicos 2000-2016

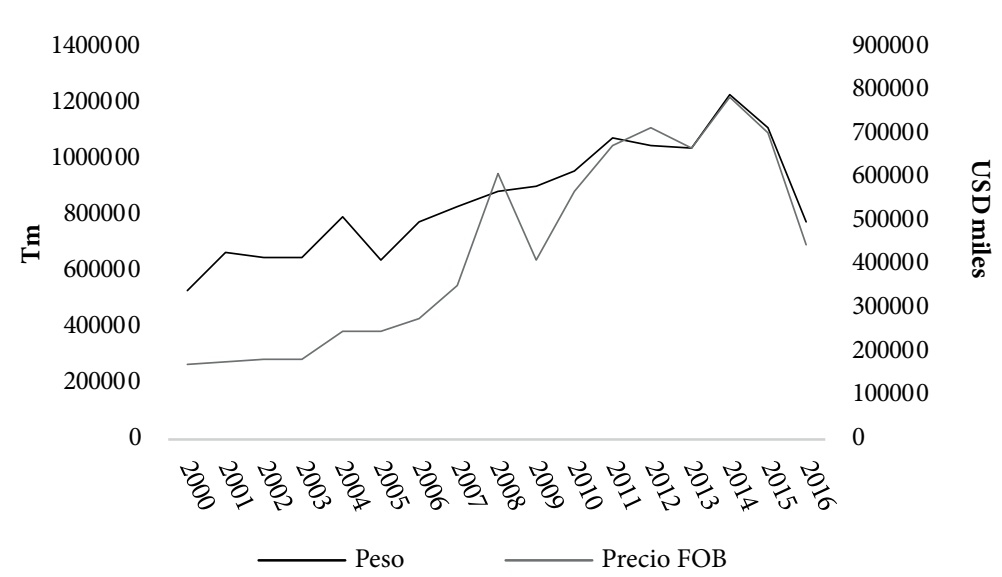

Fuente: Banco Central del Ecuador (2016). Elaboración: propia.

Figura 3b. Importacion de semillas 2000-2016

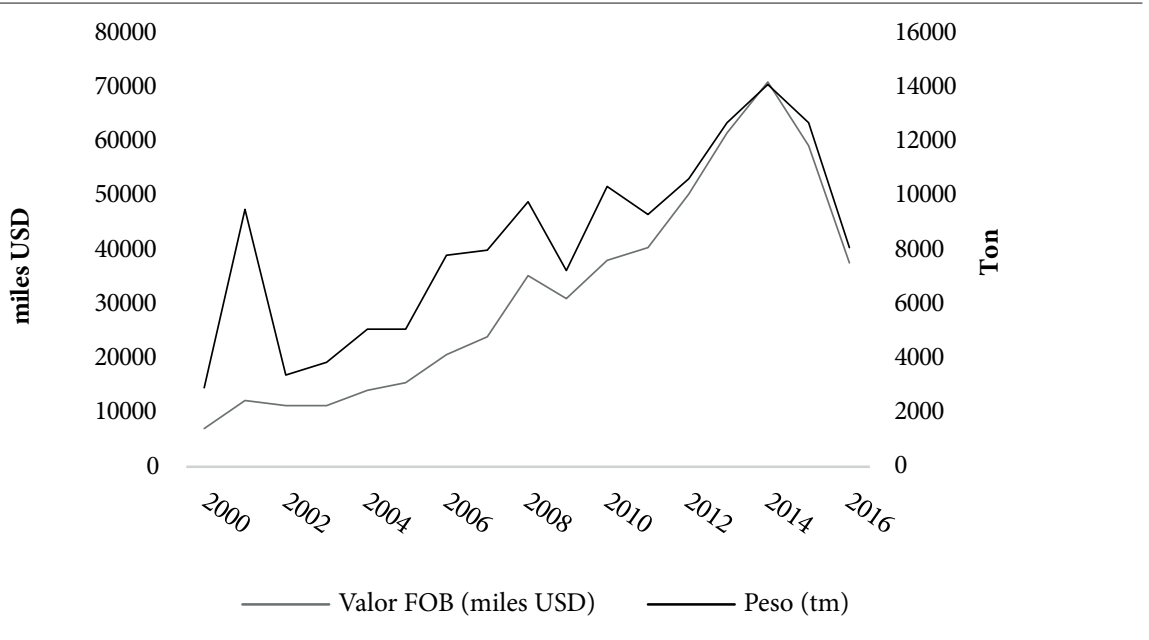

Fuente: Banco Central del Ecuador (2016). Elaboración: propia.

y agroquímicos; y el Programa Nacional de Semillas para Agrocadenas Estratégicas (PNSAE). De acuerdo al último Censo Nacional Agropecuario (INEC, 200o), al año 2000 existían 684.222 unidades productivas agropecuarias (UPAS) produciendo cultivos permanentes y transitorios en 1.763 .282 hectáreas. De estas UPAS, 239,871 (35,06\%) estaban destinadas a la producción de commodities - banano, café, cacao, palma africana, maíz duro, caña de azúcar, y plátano- sobre 52,93\% de esta superficie. Al analizar la estratificación por superficie y número de upas de los commodities en expansión, se observa que hay un alto porcentaje de pequeños 
y medianos productores, y que en el caso del maíz, café y cacao son la mayoría (ver Tabla 2). En el caso del banano y la palma africana, si bien también hay un porcentaje importante de pequeños $-79,1 \%$ y $60,2 \%$ respectivamente- y medianos productores $-13,2 \%$ y $21,4 \%$ respectivamente-, la mayor cantidad de tierra se encuentra en los grandes productores $-65,4 \%$ y $46,6 \%$, respectivamente-.

En el caso de la palma africana, de acuerdo a la Asociación de Productores de Palma (ANCUPA), al 2014 el porcentaje de pequeños productores ascendió a 87,1\%. En el caso del maíz, de acuerdo al MAG (2015), de las 104.00o UPAS, 92,500 sería productores con menos de 10 ha $-88,4 \%$ - En el caso del cacao y café, no se tienen cifras actualizadas, sin embargo, dada la promoción que el Programa Reactivación de la producción de Café y Cacao, se estima que estos números también se han incrementado.

Se constata así que una importante cantidad de mano de obra campesina está orientada a la producción de commodities, reemplazando cultivos diversificados destinados a su propio abastecimiento y a la provisión nacional de alimentos. Este cambio de cultivos viene acompañado de un cambio en la forma de producir, fomentado por el modelo modernizador que impulsa el uso del kit tecnológico en nombre del incremento de la productividad, la erradicación del hambre y la pobreza, como se observa a continuación.

\section{MODERNIZACIÓN: DEPENDENCIA E INSUSTENTABILIDAD}

Como se describió anteriormente, el PNSAE, se crea con el objetivo de incrementar la productividad en cadenas estratégicas para beneficiar a 136.00o pequeños y medianos productores, reduciendo costos de producción y garantizando disponibilidad, acceso y uso tecnificado de semillas de alto rendimiento, según lo expuesto por el Ministerio de Agricultura (MAG, 2016). La semilla certificada de alto rendimiento viene acompañada de fertilizantes, herbicidas y plaguicidas, que permiten que esta semilla potencialice su rendimiento. Este combo es conocido como el kit tecnológico.

Más del 90\% de la inversión y esfuerzos del PNSAE se han destinado al maíz y arroz, sin embargo, otros productos ${ }^{6}$ también han sido beneficiados por este o por proyectos anteriores. Actualmente, el 64\% del maíz, 24\% del arroz, 75\% de la caña de azúcar, 3\% de la papa, 0,05\% del frejol seco, o, $44 \%$ de maíz suave y $18 \%$ de hortalizas se siembran con semilla certificada (MAG, 2016).

Los principales beneficiarios de este programa son las casas comerciales que suministran los kits tecnológicos, ya que aproximadamente el 85,9\% del presupuesto del proyecto - USD 211,2 millones- es destinado al subsidio para la obtención del kit tecnológico (ibid.), valor pagado directamente a las casas comerciales. El subsidio que cubre el MAG asciende aproximadamente al 50\% del costo, con lo cual un monto similar es el que han pagado los productores a las casas comerciales. Grosso modo, en los cuatro años de duración del proyecto, las casas comerciales han recibido alrededor de UsD 360 millones.

Este modelo dependiente de insumos externos se evidencia con el incremento en la importación de semillas y de agroquímicos. Entre 2000 y 2015 la cantidad de semillas importadas se multiplicó por cinco y su costo por ocho, mientras la cantidad de agroquímicos importada se duplicó y su costo se multiplicó por seis (ver Figuras za y b). 
Tabla 03. Productos que han incrementado su consumo percápita (kg/persona/año)

\begin{tabular}{lccccccc}
\hline PRODUCTO & $\mathbf{1 9 6 1}$ & $\mathbf{1 9 7 0}$ & $\mathbf{1 9 8 0}$ & $\mathbf{1 9 9 0}$ & $\mathbf{2 0 0 0}$ & $\mathbf{2 0 0 7}$ & $\mathbf{2 0 1 3}$ \\
\hline Arroz & 21,34 & 23,72 & 30,35 & 42,67 & 55,48 & 43,78 & 45,07 \\
\hline Trigo & 20,59 & 20,82 & 37,62 & 37,27 & 38,3 & 35,46 & 39,19 \\
\hline Cerveza & 8,4 & 10,73 & 22,77 & 13,55 & 13,02 & 24,48 & 38,65 \\
\hline Carne de aves de corral & 1 & 1,54 & 2,56 & 6,92 & 15,19 & 23,74 & 21,85 \\
\hline Carne de vaca & 8,02 & 10,28 & 9,32 & 9,88 & 13,64 & 16,41 & 16,61 \\
\hline Carne de cerdo & 6,49 & 4,97 & 7,91 & 6,98 & 9,07 & 13,53 & 14,44 \\
\hline Aceite de palma & 3,64 & 3,54 & 4,49 & 9,92 & 10,13 & 9 & 8,19 \\
\hline Huevos & 1,6 & 1,74 & 5,3 & 4,82 & 4,5 & 5,26 & 8,01 \\
\hline Aceite de soya & 0,67 & 1,79 & 3,14 & 4,68 & 2,94 & 4,33 & 5,48 \\
\hline Manzanas & 0,47 & 0,86 & 4,05 & 3,22 & 2,14 & 4,41 & 4,31 \\
\hline
\end{tabular}

Fuente: FAOSTAT (2015). Elaboración: propia.

Tabla 04. Productos alimenticios que han disminuido su consumo percápita.

\begin{tabular}{lccccccc}
\hline & $\mathbf{1 9 6 1}$ & $\mathbf{1 9 7 0}$ & $\mathbf{1 9 8 0}$ & $\mathbf{1 9 9 0}$ & $\mathbf{2 0 0 0}$ & $\mathbf{2 0 0 7}$ & $\mathbf{2 0 1 3}$ \\
\hline Bananos & 122,02 & 119,42 & 97,41 & 55,61 & 129,92 & 60,18 & 35,49 \\
Plátanos & 54,31 & 57,38 & 73,11 & 74,73 & 27,73 & 32,41 & 23,97 \\
Hortalizas, otras & 51,29 & 39,42 & 22,64 & 22,17 & 17,2 & 17,15 & 20,47 \\
Patatas & 46,5 & 66,39 & 32,87 & 26,66 & 10,48 & 19,18 & 19,33 \\
Naranjas, mandarinas & 28,23 & 24,5 & 64,22 & 8,82 & 12,02 & 5,91 & 5,05 \\
Yuca mandioca & 24,97 & 24,07 & 17,7 & 9,93 & 5,6 & 0,71 & 2,53 \\
Maíz & 21,53 & 28,03 & 8,48 & 7,68 & 6,1 & 7,21 & 8,3 \\
Cebada & 8,76 & 6,64 & 2,15 & 0,95 & 1,44 & 0,01 & 0 \\
Limones, limas, cítricos & 11,06 & 6,11 & 3,12 & 6,5 & 3,54 & 1,84 & 1,78 \\
Batatas, boniatos & 4,03 & 1,48 & 0,29 & 0,34 & 0,07 & 0,19 & 0,12 \\
Tomates & 4,01 & 3,61 & 4,34 & 7,82 & 5,28 & 5,22 & 1,8 \\
Frijoles, legumbres & 10,81 & 11,03 & 4,61 & 3,39 & 4,08 & 2,42 & 2,05 \\
\hline
\end{tabular}

Fuente: FAOSTAT (2015). Elaboración propia.

Se puede pensar que el incremento en la importación de agroquímicos se debe al incremento de la superficie cultivada. Sin embargo, lo que se observa es que entre el 2002 y el 2014, la cantidad de fertilizantes por hectárea se incrementó en un 148\% y su costo en un 500\%. En los fitosanitarios, la cantidad por hectárea se incrementó en un $167 \%$ y el costo en un $252 \%$ de acuerdo a datos del Banco Central del Ecuador (BCE, 2016) y el INEC (2016). 
Como se puede observar, se está promoviendo un modelo dependiente a las importaciones, que reproduce la tendencia global del territorio capitalista y produce, a través de la dependencia externa, la desterritorialización de la soberanía alimentaria y del campesinado, además de las repercusiones ambientales y en la salud que el elevado uso de agroquímicos genera.

Los aspectos expuestos hasta aquí tienen un efecto a su vez en otros aspectos claves cuando hablamos de soberanía alimentaria, los cuales se analizan a continuación.

\section{DEPENDENCIA EXTERNA}

Otros dos aspectos importantes a explorar en relación al metabolismo del sistema agroalimentario bajo la mirada de la soberanía alimentaria son: i) la dependencia alimentaria, es decir, qué tanto dependemos de la importación de productos y cómo ha evolucionado esta dependencia, y ii) qué está comiendo la población y cómo ha evolucionado su patrón de consumo.

Si cada vez más tierra está orientada a la producción de commodities para la exportación, es evidente que la producción exportada también ha incrementado; sin embargo, es menos obvio qué implicaciones tiene esto en la cantidad de productos importados. En el año 1961, el Ecuador exportaba el 10\% de su producción, al 1990 se incrementó al 17\%; sin embargo, en los siguientes 23 años el porcentaje prácticamente se duplicó, exportándose para el 2013 el 33\% de la producción (FAOSTAT, 2015). El porcentaje de productos importados para el abastecimiento interno también ha venido incrementado. En 1961 se importaba el 1\% de la demanda interna de alimentos, al 1990 este porcentaje se incrementó al 4\%, y ya para el 2013 el porcentaje ascendió al 9\%, más del doble en 23 años. Los grupos alimenticios cuya importación representa más del 50\% del consumo interno son las legumbres secas, pescado y frutos del mar y estimulantes; en menor cantidad cereales, hortalizas y frutas.

Al observar la evolución del sector externo del sistema agroalimentario, se evidencia una incoherencia en cuanto al destino de la producción de alimentos, cuya creciente exportación vincula a su vez la exportación de la tierra, mano de obra campesina y demás recursos productivos implicados en su producción, ${ }^{7}$ y a la consecuente dependencia externa de productos de los cuales en años anteriores el país era autosuficiente.

\section{PATRONES DE CONSUMO Y SALUD DE LA POBLACIÓN AL SERVICIO DE LA ACUMULACIÓN}

Los consumidores constituimos los otros actores fundamentales para facilitar la acumulación de los grupos económicos a nivel nacional e internacional. Es preciso moldear el consumo de la gente de tal manera que sirva a este propósito. Vemos así que coincidentemente productos como arroz, trigo, leche, azúcar, aceites de palma y soya y carnes de cerdo y aves de corral, productos vinculados con los grupos económicos anteriormente revisados, han incrementado considerablemente su consumo per cápita Uno de los cambios más conspicuos se observa en el consumo de aves de corral, el cual en 52 años se incrementó 19 veces (ver Tabla 3). Al contrario, productos como el maíz, bananos, plátanos, papas, yuca, cebada y ciertas legumbres, vinculados mayormente con la producción campesina y que anteriormente aportaban con el 35,5\% del consumo per cápita, ahora aportan únicamente el 10\% (ver Tabla 4). 
Este cambio en los patrones de consumo, principalmente el incremento en el consumo de carbohidratos como el arroz y el trigo, de aceite de palma y azúcar, están teniendo consecuencias graves en la salud de la población. Actualmente el sobrepeso y ligado a éste las enfermedades no transmisibles como las cardíacas y la diabetes, ha incrementado a tal punto de convertirse en la primera causa de muerte en el Ecuador (Freire, et al., 2014). Este patrón sigue la tendencia global; la Organización Mundial de la Salud (oms, 2018) sostiene que la obesidad ha alcanzado niveles considerados epidémicos. ${ }^{8}$. A este problema le acompaña muchas veces niveles de desnutrición, constituyéndose lo que se ha denominado la doble carga de la malnutrición, que en el caso del Ecuador tiene un costo para el Estado de USD 4300 millones - 4,3\% del PIB- de acuerdo al informe presentado por la Comisión Económica para América Latina y el Caribe y el Programa Mundial de Alimentos (CEPAL y PMA, 2017).

\section{CONCLUSIONES}

El metabolismo del sector agroalimentario es producido por diversas estrategias de poder y es servil al proceso de acumulación de las élites económicas que lideran el sector. Los fondos esenciales para garantizar la sostenibilidad del sistema agroalimentario y garantizar la soberanía alimentaria están siendo orientados a la producción de commodities, bajo un modelo que demanda cada vez más insumos externos, creando un modelo dependiente de materiales y energía. Los flujos alimentarios vinculados con el sector externo también obedecen a este modelo incoherente que exporta y al mismo tiempo importa cada vez más cantidades de alimentos, y con éstos se exporta e importa tierra, agua y energía, generando un modelo insostenible.

Por otro lado, además del campesinado, los consumidores son serviles a estos procesos de acumulación gracias a estas estrategias de poder que moldean prácticas productivas y patrones de consumo.

Analizar la integralidad del sistema agroalimentario bajo la óptica de la economía ecológica y la ecología política evidencia que las dinámicas de poder están arraigadas y forman parte de la estructura institucional que rige el sistema agroalimentario a través de distintas estrategias. Esta realidad constituye un impedimento al momento de pensar la construcción y aplicación de una política pública que procure una real sustentabilidad del sistema agroalimentario y la soberanía alimentaria. Al mismo tiempo, este conocimiento permite plantear la urgencia de una propuesta política con una real voluntad para reconstruir, desde la estructura, la institucionalidad y colocar al Estado al servicio de la población, de su Buen Vivir y que viabilice la garantía de la SA. Por otro lado, nos brinda la oportunidad de plantear procesos de resistencia y de transición a modelos alternativos de manera realista y estratégica.

Sin embargo, para lograr esto último es imprescindible también que estos conocimientos y análisis trasciendan el ámbito académico, que se lleven a espacios de discusión y construcción colectiva, que involucre a diversos actores sociales de las clases subalternas, desposeídas, y en resistencia, que son los actores centrales de la ecología política, pero también a espacios de la sociedad civil en general, de las clases medias despolitizadas, que son un sector clave para viabilizar procesos de transición a modelos alternativos al capitalismo. 


\section{NOTAS}

1 Mercancías, productos producidos y gestionados bajo la lógica del mercado.

2 Firmado el 8 de agosto del 2002.

3 Entre los cultivos permanentes, hay palma africana, cacao, café; mientras que en los cultivos semipermanentes existen el banano, caña de azúcar, palmito.

4 Información obtenida del mapa Mapa de zonificación paisajística y capacidad de acogida elaborado por el Instituto Espacial Ecuatoriano (IEE, 2015).

5 El sector de los balanceados es manejado mayormente por el grupo económico PRONACA, el más grande del sector agroalimentario.

6 Desde 2016 se promueve el uso de semilla certificada de quinua, amaranto, cebada, frejol, haba, papa, arveja, chocho, trigo, algodón, maíz suave, cítricos, uvilla, fresa, naranjilla, mora, mango, aguacate, cítricos, tomate de árbol, granadilla, tomate riñón, hortalizas, cebolla, caducifolios, caña de azúcar.

7 Para un detalle de los recursos inmersos en la exportación de productos alimenticios ver la tesis doctoral El uso de la gramática del MusIASEM para el análisis cuantitativo de la sostenibilidad de los sistemas alimentarios, de Juan Cadillo (2015).

$8 \mathrm{Al}$ 2016, 1,9 mil millones de adultos mayores de 18 años tienen sobrepeso; de éstos, 650 millones son obesos y 340 millones de niños, niñas y adolescentes tienen sobrepeso o son obesos.

\section{REFERENCIAS}

Alier, J. M. (1999). Introducción a la Economía Ecológica. Madrid, España. Editorial Traficantes de Sueños. Ashley, J. (2010) African oil palm: impacts in Equador's Amazon. Cultural Survival. Recuperado de http://www.culturalsurvival.org/publications/cultural-survival-quarterly/ecuador/ african-palm-oil-impacts-equadors-amazon

BCE. (2015). Estadísticas de importación de semillas y agroquímicos. Quito, Ecuador.

Buitrón, R. (2001). El caso de Ecuador: ¿El paraíso en siete años? En R. Carrere (Coord.), El amargo fruto de la palma aceitera: despojo y deforestación. (pp. 20-27). Montevideo, Uruguay: Movimiento Mundial por los Bosques Tropicales.

CEPAL y PMA. (2017). El costo de la doble carga de la malnutrición. Recuperado de http://es.wfp.org/sites/ default/files/es/file/espanol_brochure_26_abril_2017.pdf.

Estevan, A., Jover, D. y Naredo, J.M. (2009). Por una economía ecológica y solidaria. Conversaciones con Antonio Estevan y José Manuel Naredo. Barcelona, España: Icaria Editorial.

Foucault, M. (2014). Las redes del poder. Buenos Aires, Argentina: Prometeo libros.

Freire W., Ramírez-Luzuriaga M., Belmont P., Mendieta M., Silva-Jaramillo M., Romero N., Sáenz K., Piñeiros P., Gómez L., Monge R. (2014). Tomo I: Encuesta Nacional de Salud y Nutrición de la población ecuatoriana de o a 59 años. ENSANUT-ECU 2012. Quito, Ecuador: Ministerio de Salud Pública/Instituto Nacional de Estadísticas y Censos.

Georgescu-Roegen, N. (2007). Hacia una economía humana. En N. Georgescu-Roegen. Ensayos Bioeconómicos, (pp. 31-34). Madrid, España: Los Libros de la Catarata.

Georgescu-Roegen, N. (2007). La crisis de los recursos naturales. En N. Georgescu-Roegen. Ensayos Bioeconómicos, (pp. 87-94). Madrid, España: Los Libros de la Catarata.

Georgescu-Roegen, N. (2007). La ley de la entropía y el problema económico. En N. Georgescu-Roegen. Ensayos Bioeconómicos, (pp. 35-52). Madrid, España: Los Libros de la Catarata.

Giampetro, M. y Bukkens, S. (2014) Analysis of societal and ecosystem metabolism. En M. Giampetro, R. 
Aspinall, J. Ramos-Martin y S. Bukkens (Eds.). Resource Accounting for Sustainability Assessment, (pp. 11-21). (s. d.).

Giampetro, M. y Lomas, P. (2014). The interfas between societal and ecosystem metabolism. En M. Giampetro, R. Aspinall, J. Ramos-Martin y S. Bukkens (Eds.), Resource Accounting for Sustainability Assessment, (pp. 33-48). (s. d.).

Herrero, Y. (2015). Una economía al servicio de la vida y dentro de los límites físicos de la tierra. En Y. Álvarez, A. Encinas, C. Saavedra, M. Olcina e I. Jiménez (Eds. y Coord.). Economía de Escalera, finanzas de patio. El salmón contracorriente. Recuperado de http://www.elsalmoncontracorriente. es/?Una-economia-al-servicio-de-la

INEC. (2001). III Censo Nacional Agropecuario. (s. d.).

INEC. Encuesta de producción agropecuaria continua 2002-2014. Recuperado de http://www.ecuadorencifras.gob.ec

Kay, C. (December, 2015). The agrarian question and the neoliberal rural transformation in Latin American. European Review of Latin American and Caribean Studies, (100), 73-83.

Massey, D. (1993). Power geometry and a progressive sense of place. En B. Jon, B. Curtis, T. Putnam, G. Roberston y L. Tickner (Eds.), Mapping the futures. Local cultures, global change, (pp. 59-69). London, UK: Routledge.

McMichael, P. (2009). A food regime genealogy. Journal of Peasant Studies, 36(1), 139-169. Dor: 10.1080/03066150902820354

MAG. (2016). Ficha informativa de proyecto 2016: Proyecto Nacional de Semillas para agrocadenas estratégicas. Recuperado de http://servicios.agricultura.gob.ec.

MAG. (2015). Aplicación de la metodología: Monitoreo y Análisis de Políticas Agrícolas y AlimentariasMAfap, caso Maíz Amarillo duro. [Presentación proporcionada por la Subsecretaría de Comercialización].

MAG. (2014). Mapa de cobertura y uso de la tierra 2008-2014. Recuperado de http://sni.gob.ec/ mapa-cobertura-uso.

Ministerio del Ambiente. (2008). Mapa de cobertura y uso de la tierra 2008. Recuperado de http://mapainteractivo.ambiente.gob.ec.

OMs. (2018, febrero 16). Obesidad y sobrepeso. Datos y cifras. Recuperado de http://www.who.int/es/ news-room/fact-sheets/detail/obesity-and-overweight

Pastor, C. (2016). Los grupos económicos en el Ecuador. Quito, Ecuador: Ediciones La Tierra.

Van Hauwermeiren, S. (1999). Manual de Economía Ecológica. Quito, Ecuador. Editorial Abya-Yala.

Yumbla, M. R. (agosto, 2014). Agricultura bajo contrato con intervención estatal. Estudio de caso: Maíz duro cantones Palenque y Pedro Carbo-Ecuador. Reporte Técnico. Avsf. Quito, Ecuador.

Zapata, A., Ruíz, P. y Brassel, F. (2008). La estructura agraria en el Ecuador: una aproximación a su problemática y tendencias. En F. Brassel, S. Herrera, y M. Laforge, (Eds.), ¿Reforma Agraria en el Ecuador?: viejos temas, nuevos argumentos, (pp. 17-32). Quito, Ecuador: SIPAE. 
